\title{
RADIO EMISSION FROM THE DIRECTION OF THE SUPERGALAXY
}

\author{
J. E. BALDWIN AND J. R. SHAKESHAFT \\ Mullard Radio Astronomy Observatory, Cavendish Laboratory \\ Cambridge, England
}

Kraus and Ko [1] and Hanbury Brown and Hazard [2] have suggested that the band of radio emission running roughly perpendicular to the galactic plane at about $12^{\mathrm{h}}$ right ascension, represents the integrated emission from the concentration of bright galaxies lying along a great circle that crosses the galactic plane at longitudes $l=105$ degrees and 285 degrees. This paper puts forward certain difficulties in this interpretation. These galaxies and our own are believed by de Vaucouleurs $[3,4]$ to be members of a "cluster of clusters," which he terms the local supergalaxy, after Shapley.

An examination of the brightness-temperature contours that have been derived by Baldwin from a new survey of the background radiation at $159 \mathrm{Mc} / \mathrm{s}$ (Fig. 1) reveals that the detailed correlation between galactic density and radio brightness is poor. It may be noted for example that the densest concentration of galaxies, the Virgo cluster, lies in a dip of the contours. In addition, there are more intense emission regions nearby that are not associated with any optical features. In his introduction to the session on radio sources Hanbury Brown drew similar conclusions from a survey of this region with the Jodrell Bank 250-foot paraboloid.

The supergalaxy is said to include galaxies down to the 18th magnitude but a study of galactic counts made by Shapley and Jones to $m_{p q} \Rightarrow 17.5$ for a strip centered on declination +43.5 [5] shown that the correlation with faint galaxies is no better.

The radio radiation expected from the normal galaxies in the supergalaxy is less than 20 per cent of that observed. The excess cannot be accounted for by abnormal galaxies, which would appear individually as extra point sources but are not in fact detected.

Shklovskii [6] has proposed that the emission is caused by relativistic electrons accelerated by intergalactic magnetic fields throughout the whole volume of the supergalaxy. In this case, dense clusters such as those in Coma and Perseus might be expected to be especially strong sources. The radiation due to the normal galaxies in these clusters cannot be estimated accurately, but the observed flux densities do not seem to be much greater than would be expected from the normal galaxies alone.

De Vaucouleurs further suggests that there are other supergalaxies near the local one, the nearest one appearing as an elliptical swarm of galaxies near $l=155$ degrees, $b=-40$ degrees. If excess radiation is associated with 


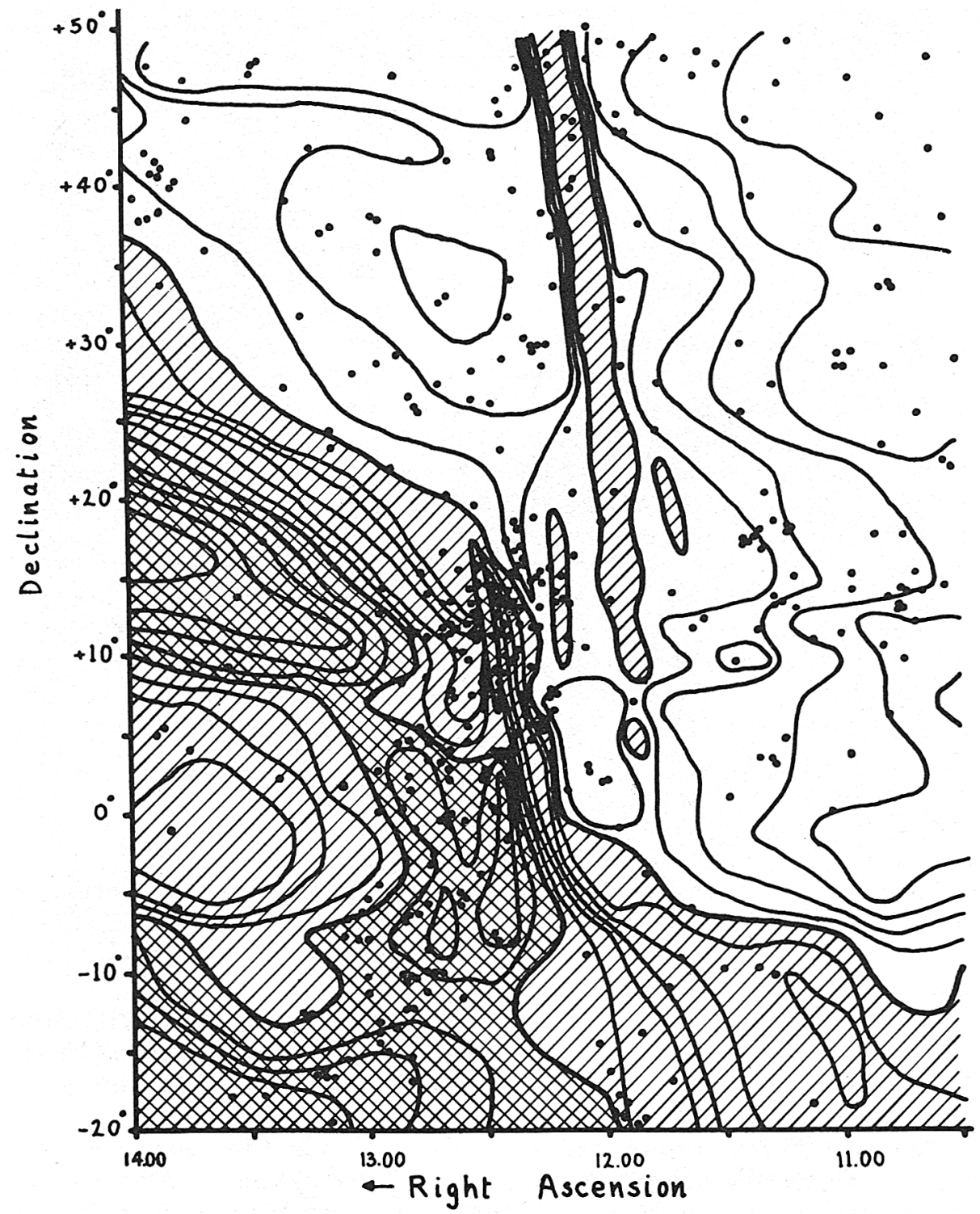

Fig. 1. Contours of aerial temperature at $159 \mathrm{Mc} / \mathrm{s}$ plotted together with galaxies from the Shapley-Ames catalog. The contour interval represents $8^{\circ} \mathrm{K}$ of aerial temperature and the hatched regions are the more intense. The main features to be noted are: $(a)$ The general increase in intensity from $10^{\mathrm{h}} 30^{\mathrm{m}}$ to $13^{\mathrm{h}}$ associated with the galactic background. (b) The intense ridge between $+08^{\circ}$ and $+27^{\circ}$ and extending from $12^{\mathrm{h}} 30^{\mathrm{m}}$ to $14^{\mathrm{h}}$. This is believed to be galactic. (c) A bright region between $-15^{\circ}$ and $+05^{\circ}, 12^{\mathrm{h}} 15^{\mathrm{m}}$ to $13^{\mathrm{h}}$. This is the feature that Kraus and Ko associated with the supergalaxy. (d) A comparatively faint ridge between $11^{\mathrm{h}}$ and $12^{\mathrm{h}} 30^{\mathrm{m}}$ at declinations north of $+15^{\circ}$. Part of this feature was studied by Hanbury Brown and Hazard.

[N.B. The narrow ridge at $12^{2} 30^{\mathrm{m}},+12^{\circ}$ is due to the intense source Virgo $\mathrm{A}$, and that running north from $+10^{\circ}$ at $12^{\mathrm{b}}$ due to the source Cassiopeia A.] 
supergalaxies then it too should be easily detectable in the surveys of Bolton and Westfold [7] and of Baldwin, but no trace of it can be found.

Perhaps the most serious difficulty is that the emission from the direction of the supergalaxy $\left(20\right.$ to $\left.80^{\circ} \mathrm{K}\right)$ is comparable with the total isotropic extragalactic component, to which an upper limit has been set by Baldwin [8], which at $159 \mathrm{Mc} / \mathrm{s}$ is equivalent to $90^{\circ} \mathrm{K}$. On the hypothesis, therefore, that this radiation is physically associated with the local supergalaxy, the first 15 megaparsecs (the extent of the supergalaxy, according to de Vaucouleurs) measured from the earth along a line of sight that intersects the Supergalaxy must be intrinsically different from, say, the next 1000 megaparsecs. In view of the large-scale uniformity found among the more distant galaxies this does not seem likely.

These arguments do not support the connection of the radio feature with the local supergalaxy and it may be that it originates within the Galaxy. This suggestion is made more plausible by the presence of the nearby intense belt of emission (discussed by F. G. Smith in connection with paper 102), which is almost certainly galactic.

\section{REFERENCES}

[1] Kraus, J. D., and Ko, H. C. Nature, 172, 538, 1953.

[2] Brown, R. Hanbury, and Hazard, C. Nature, 172, 997, 1953.

[3] de Vaucouleurs, G. A.J. 58, 30, 1953.

[4] de Vaucouleurs, G. Vistas in Astronomy. London (Pergamon Press), 1956, Vol. II.

[5] Shapley, H., and Jones, R. B. Proc. Nat. Acad. Sci., Wash., 26, 554, 1940.

[6] Shklovskii, I. S. A. Zh. 31, 533, 1954.

17] Bolton, J. G., and Westfold, K. C. Aust. J. Sci. Res. A 3, 19, 1950.

[8] Baldwin, J. E. M.N.R.A.S. 115, 690, 1955.

\section{Discussion}

Pawsey: Eric Hill in Sydney has also failed to identify the "supergalaxy" using $85 \mathrm{Mc} / \mathrm{s}$ Mills cross contours in the region south of declination +10 degrees. The contours in this region appear independent of the disposition of the galaxies: either the supergalaxy does not emit appreciably or, if it does, it is obscured by other features.

Hoyle: I should like to express Shakeshaft's conclusion on magnetic fields within the so-called supergalaxy in somewhat different form. Instead of saying there is no such magnetic field, I think it is preferable to say that the magnetic field is not systematically higher within the supergalaxy than anywhere else.

Shakeshaft: Any radio emission by the synchrotron mechanism from intergalactic regions will of course depend on the cosmic ray electron density, which is there unknown, so that the radio observations do not eliminate the possibility of intergalactic magnetic fields.

Westerhout: I should like to make a few comments connected with the previous papers. In the first place, I should like to draw attention to the 
excellent agreement between the Harvard and Dwingeloo contours of the Cygnus region at $1390 \mathrm{Mc} / \mathrm{s}$. At the same time it is clear that even a factor 1.5 improvement in beamwidth gives quite a bit more resolution (Harvard 49 and Dwingeloo 34 minutes of arc). The fact that with the narrow beam Cygnus $\mathrm{X}$ is now resolved into many sources shows the danger of deciding on the character of an extended source measured with a wide beam. So far, Cygnus $\mathrm{X}$ has been in general assumed to be one large source. There are two sources, IC 443 and the Cygnus loop, both nonthermal at the lower frequencies, that show the influence of the thermal component at $1390 \mathrm{Mc} / \mathrm{s}$ in a displacement of the center and a flattening-out of the spectrum. A source that has been identified with $\mathrm{M} 20$ in many catalogs is now shown to be nonthermal and about 0.5 degrees southwest of $M 20$.

Hazard: I would like to say a few words about the radiation from the region of the supergalaxy, since at Jodrell Bank we now have very extensive observations at 90 and $160 \mathrm{Mc} / \mathrm{s}$. The $90-\mathrm{Mc} / \mathrm{s}$ survey covers a range of north declinations from 70 to 30 degrees and the $160-\mathrm{Mc} / \mathrm{s}$ survey from 60 to 20 degrees. In the region from 70 to 30 degrees there is a good general correlation between the bright extragalactic nebulae and the observed band of radio radiation, but the detailed correlation is not good. Below 30 degrees the correlation is not good and there is no clear evidence of an association between the radio observations and the concentration of extragalactic nebulae. Probably the most interesting feature is that the $90-\mathrm{Mc} / \mathrm{s}$ records with their high signal-to-noise ratio show clear evidence of resolution of the radio band into a number of discrete sources. These do not appear on an interferometer record of the same region, and since the lobe-spacing of the interferometer was 22 minutes of arc, this means that the sources are of large angular diameter. Further work indicates that they are probably of the order of one degree in diameter. It therefore appears that at least part of the intensity in the strip consists of radiation from a small number of large-diameter sources. While I do not wish to assert that the radio isophotes and the distribution of the extragalactic nebulae are related, I would point out that if the radiation does consist of a small number of sources, a detailed correlation between the radio and optical observations would not be expected even though the two were associated.

Edmondson: The current views of Dr. Harlow Shapley, who originated the term supergalaxy, might well be recorded here.

Shapley (communication to the Editor): The first use of the term "supergalaxy" was, I believe, when I suggested that the cloud of bright galaxies in Virgo constituted a physical organization greater in extent and mass than the largest single or double galaxy. Because of the evidence of a local system of stars, the equatorial plane of which is inclined many degrees (perhaps 10 degrees) to the galactic plane (Harvard Circular 229, 1922), and because of the early indication that our Milky Way system is much larger than an average galaxy, I suggested in Harvard Circular 350, 1930, that our Milky Way organization also might be composite and made up of several intermingling galaxies; for instance, made up of the local star system, the Cygnus 
star cloud, the Sagittarius star clouds, perhaps others, and perhaps also containing the Magellanic Clouds, although they lie well away from the average equatorial plane.

Now we know that the local system and other star clouds of the Milky Way are spiral-arm details of our own giant Galaxy, and that the Magellanic Clouds are in a local group of galaxies, but not closely involved with the Milky Way. Our Milky Way, therefore, is not a supergalaxy.

With the completion of the Shapley-Ames catalog of the thousand brightest galaxies, it was seen, as one detail of nearby metagalactic structure, that the Virgo cloud extends southward at least to the bounds of Centaurus, and northward through Coma, and into Ursa Major and other constellations. It is indeed a sort of supersystem, shown clearly in the plots of Harvard Annals, 88, No. 2-plots that have been reproduced several times since their original publication. The Lund workers on galaxy distribution also called attention to these streams of bright galaxies and noted that in a sense they constituted an elongated cluster of clusters of galaxies. The term supergalaxy is here appropriate if the terms cloud or cluster are not deemed sufficient.

On various occasions I have pointed out that it seems farfetched to assume that in the distribution of bright galaxies we have evidence of a basic metagalactic plane, with respect to which one could and should associate metagalactic latitude and longitude. That a group of a thousand or so galaxies, in a volume some ten million light years in diameter, should dominate the distribution of a billion galaxies, spread throughout a volume ten billion light years in diameter, is more than unlikely. The local arrangement should not encourage over-all metagalactic model making.

In the early photographic study of globular clusters some enthusiastic workers saw in them clear indications of spiral structure. When a cluster of a hundred or so stars is considered, it is easy to trace out spiral lines, if the number, pitch, center, and richness of the spiral arms can be selected to suit the assumptions. Longer exposures, showing thousands of stars, quickly disposed of the spiral hypothesis for globular clusters, for the "arms " promptly disappeared with the increase in numbers of stars. Similarly, deeper surveys of galaxies tend to wash out the appearance of a unique supergalaxy of significant consequence in metagalactic dynamics.

At best we can use the term "local supergalaxy" when referring to the Ursa Major-Virgo-Centaurus cloud, or to similar aggregations that are shown in the Harvard, Lick, and Palomar surveys. Certainly these local features do not control over-all metagalactic structure. Perhaps we should not include as a part of the Virgo distributional irregularity the Fornax group and other bright galaxies on the south side of the galactic plane.

The term "supergalaxy" has not, I believe, been used in reference to single giant galaxies like M 31 and the brightest members in clusters of galaxies. Apparently, as revealed by our revised distance scale for objects outside our Galaxy, there are in the remote clusters of galaxies individuals much brighter and bigger than our own. But for them the term giant should suffice. 